\title{
Parameter estimation in kinetic reaction models using nonlinear observers is facilitated by model extensions *
}

\author{
Dirk Fey*, Rolf Findeisen ${ }^{* *}$, Eric Bullinger* \\ * Industrial Control Centre, University of Strathclyde, Glasgow, United \\ Kingdom (e-mail: dirk.fey@eee.strath.ac.uk; \\ eric.bullinger@eee.strath.ac.uk) \\ ** Institute for Systems Theory and Automatic Control, University of \\ Stuttgart, Germany, (e-mail: findeise@ist.uni-stuttgart.de)
}

\begin{abstract}
An essential part of mathematical modeling is the accurate and reliable estimation of the model parameters. In biology, the required parameters are particularly difficult to measure because of either shortcomings of the measurement technology or a lack of direct measurements. In both cases, parameters must be estimated from indirect measurements, usually in the form of time-series data. Here, we present a novel approach for parameter estimation that is particularly tailored to biological models consisting of nonlinear ordinary differential equations. By assuming specific types of nonlinearities common in biology, such as generalized mass action, Hill kinetics and products thereof, we can take a three step approach: (1) transform the identification into an observer problem using a suitable model extension that decouples the estimation of non-measured states from the parameters; (2) reconstruct all extended states using nonlinear observers; (3) estimate the parameters using the reconstructed states. The actual estimation of the parameters is based on the intrinsic dependencies of the extended states arising from the definitions of the extended variables, and solved via least squares approximation. An important advantage of the proposed method is that it allows us to identify suitable measurements and/or model structures for which the parameters can be estimated. In addition, the proposed identification approach is generally applicable to models of metabolic networks, signal transduction and gene regulation.
\end{abstract}

Keywords: parameter estimation, parameter identification, biological systems, biochemical systems, high-gain observers, reduced-order observers, observer Lyapunov function.

\section{INTRODUCTION}

In order to understand the dynamics and function of biomolecular networks, such as metabolic pathways, signal transduction and gene regulation, mathematical modeling presents an appropriate tool. These models depend crucially on kinetic parameters, whose accurate and reliable estimation still presents a bottleneck. However, recent advances in measurement technologies makes their indirect interference from time series data more and more feasible [Anguelova et al., 2007, Voit and Almeida, 2004].

The dynamics of cell biological processes are often modeled with reaction kinetic systems composed of ordinary differential equations. Thereby, the reaction rates $r$ as well as the measurements $y$ are represented by nonlinear functions that depend on the species concentrations $c$ and kinetic parameters $\rho$

$$
\frac{d c}{d t}=N r(c, \rho), \quad c(0)=c_{0} \in \mathbb{R}_{+}^{n_{c}}, \quad y=h(c, \rho) \in \mathbb{R}^{p}
$$

where $N \in \mathbb{R}^{n_{c} \times n_{r}}$ denotes the stoichiometric matrix and $c_{0}$ the initial condition for $t=0$. The solution of this

\footnotetext{
* Supported by the Science Foundation Ireland (SFI) grant 03/RP1/I382.
}

equation given a certain initial condition $c_{0}$ and parameter $\rho$ is called the trajectory of the system $c\left(t, c_{0}, \rho\right)$. From a mathematical perspective, measurements taken in an experiment somehow reflects such trajectories, and parameter estimation seeks for parameters that represent the data best based on some optimality criterion. A natural criterion, also used here, is to minimize the error between measured and simulated output $y-h\left(x\left(t, x_{0}, \rho\right)\right)$. Note that we assume continuous measurement of the output, which can for instance be achieved by interpolation of time-course measurements obtained with sufficiently large resolution.

Often Monte-Carlo based methods, evolutionary strategies or other heuristic methods are utilized. These global methods can not guarantee to find the optimal solution, are computationally expensive and require a multitude of simulation runs that in turn depend on the parameters [Moles et al., 2003]. A method that tries to minimize problematic effects arising from this circular parameter dependence is for example multiple shooting [Peifer and Timmer, 2007]. Despite undoubtedly usefulness, multiple shooting methods do however not resolve the circular dependency. Further, heuristic methods do not allow to address the question of identifiability, which asks whether 
the parameters are theoretically obtainable under the assumptions of noise free measurements and error free model [Audoly et al., 2001]. The optimum is to choose a model structure and set of measurements for which all parameters are identifiable.

Here we present an approach to resolve the circular parameter dependency within the estimation process for kinetics composed of products of generalized mass action and Hill terms. A suitable model extension eliminates the kinetic parameters in the system and establishes a one-to-one correspondence between parameters and states. This decouples the parameter and the state estimation, and further, allows us to address identifiability in terms of observability. The present manuscript is organized as follows. First some results in the field of observability are given. Second, we present the model extension together with the state and parameter estimation methods. In order to provide a proof of concept, we test the method on a model of the circadian rhythm in neurospora.

\section{PRELIMINARIES}

Before discussing observability and observers, it is useful to outline some important results in that field. For a detailed discussion of nonlinear observability considering multiple outputs we refer to Birk and Zeitz [1988].

Consider the dynamical system with output $y$

$$
\dot{x}=f(x), \quad x(0)=x_{0} \in \mathbb{R}^{n}, \quad y=h(x) \in \mathbb{R}^{p} .
$$

The observability space consists of the outputs and Liederivatives thereof

$$
\begin{aligned}
\mathcal{O}=\left\{h_{1}(x), \mathrm{L}_{f} h_{1}(x), L f^{2} h_{1}(x), \ldots,\right. & \\
& \left.h_{p}(x), \mathrm{L}_{f} h_{p}(x), L f^{2} h_{p}(x), \ldots\right\} .
\end{aligned}
$$

Note that on a trajectory $x(t)$ Lie derivatives and time derivatives are equivalent $\frac{d y}{d t}=\mathrm{L}_{f} h(x(t))=\frac{\partial h_{i}}{\partial x} f(x(t))$.

The observability map $q: \mathbb{R}^{n} \mapsto \mathcal{H}^{n} \subseteq \mathcal{O}$ is a selection of $n$ functions in that space

$$
q(x)=\left[\begin{array}{c}
q_{1}(x) \\
q_{2}(x) \\
\vdots \\
q_{p}(x)
\end{array}\right], \quad q_{i}(x)=\left[\begin{array}{c}
\mathrm{L}_{f}^{0} h_{i}(x) \\
\mathrm{L}_{f}^{1} h_{i}(x) \\
\vdots \\
\mathrm{L}_{f}^{n_{i}-1} h_{i}(x)
\end{array}\right], \quad \sum_{i=1}^{p} n_{i}=n
$$

If $q$ is smooth and has a continuous inverse ( $\hat{=}$ semidiffeomorphism) it can be used to transform the system (2) with $z=q(x)$ into observer normal form

$$
\dot{z}=\bar{f}(z), \quad z(0)=q\left(x_{0}\right), \quad y=\bar{C} z,
$$

with

$$
\bar{f}(z)=\left[\begin{array}{c}
z_{2} \\
\vdots \\
\phi_{n_{1}}(z) \\
z_{n_{1}+2} \\
\vdots \\
z_{n_{1}+\ldots+n_{p-1}+2} \\
\vdots \\
\phi_{n_{p}}(z)
\end{array}\right], \quad \bar{C}=\frac{\partial}{\partial z}\left[\begin{array}{c}
z_{1} \\
z_{n_{1}+1} \\
\vdots \\
z_{n_{1}+\ldots+n_{p-1}+1}
\end{array}\right]
$$

which is structured into $p$ modules consisting of integrator chains summarizing the nonlinearity in $\phi_{i}(z)$

$$
z_{n_{1}+\ldots+n_{i-1}+1}=y_{i}, \quad \dot{z}_{j}=z_{j+1}, \quad \dot{z}_{n_{i}}=\phi_{i}(z) .
$$

To check whether an observer exists, the system is analyzed for observability:

Definition 1. System (2) is locally observable iff the observability matrix $Q=\frac{\partial}{\partial x} q$ has full rank. It is called observable in a region $\mathcal{R} \subset \mathbb{R}^{n}$ iff it is locally observable for all $x \in \mathcal{R}$.

For observer design, often Lipschitz continuity of $\bar{f}$ is postulated, since it guarantees uniqueness of solutions of (4) given a certain initial condition. This can be relaxed, using the following definition.

Definition 2. System (2) is trajectory observable iff $q$ is a semi-diffeomorphism and (4) exhibits only one solution that generates the same output as (2) [Vargas and Moreno, 2005].

\section{METHODS}

Generally, the reaction rates in (1) may consist of any nonlinear function. For most biological models however, the kinetics possess a particular form.

Assumption 3. The reaction rates can be written as

$$
r_{i}=\hat{r}_{i} \prod_{j=1}^{n_{c}} \frac{c_{j}^{\nu_{i, j}}}{K_{i, j}^{\eta_{i, j}}+c_{j}^{\eta_{i, j}}},
$$

where the parameters are the nominal reaction rates $\hat{r}_{i} \in$ $\mathbb{R}_{+}$and the Hill-constants $K_{i, j} \in \mathbb{R}_{+}$, which describe a regulatory influence of species $j$ on reaction $i$.

Remark 4. The orders of the reactions $\nu_{i, j}$ and the Hill exponents $\eta_{i, j}$ are assumed to be known a priori, and are therefore not considered as parameters.

Remark 5. The description comprises zero order (constant reaction rates, i.e. $\left.\nu_{i, j}=\eta_{i, j}=0\right)$, mass-action kinetics $\left(\nu_{i, j} \in \mathbb{N}_{0}\right.$ and $\left.\eta_{i, j}=0\right)$, generalized mass-action $\left(\nu_{i, j} \in\right.$ $\mathbb{R}_{0,+}$ and $\left.\eta_{i, j}=0\right)$, Michelis-Menten $\left(\nu_{i, j}=\eta_{i, j}=1\right)$ as well as activating $\left(\nu_{i, j}=\eta_{i, j} \in \mathbb{R}_{0,+}\right)$ and inhibitory Hill kinetics $\left(\nu_{i, j}=0, \eta_{i, j} \in \mathbb{R}_{0,+}\right)$.

Restricting the nonlinearity in the reaction rates as above allows us to reformulate (1) into a more suitable form for addressing the identification problem. By introducing new ordinary differential equations for the reaction rates $r_{i}$ and their denominators $m_{i, j}=K_{i, j}^{\eta_{i, j}}+c_{j}^{\eta_{i, j}}$, we eliminate the dependency of the system on the parameters. These are now hidden in the initial conditions, and can be obtained by either identifying the correct initial conditions or applying a regression scheme on the extended variables. For the latter case a analytical solution minimizing the least squares criterion can be stated regardless of particular system under consideration. Thus, the problem of parameter estimation is transformed into a problem of state estimation, whereupon appropriate non-linear observers can be used.

The proposed approach can be structured into the following three steps:

(1) Transform the model into its extended form.

(2) Estimate all (non-measured) states using an appropriate (nonlinear) observer. 
(3) Infer the parameters from these state estimates through regression.

In the following, details concerning each one of these steps are presented.

\subsection{Model extension}

A crucial part of the proposed method is to reformulate the system in a parameter independent form by introducing new state variables.

Assumption 6. The concentrations $c_{i}$ and the parameters $\hat{r}_{i}$ and $K_{i, j}$ are strictly positive.

Assumption 7. Measurements consist of linear combinations of species concentrations and/or reaction rates.

Remark 8. In the context of intracellular signaling and gene regulation, measurements usually concern protein and mRNA levels, i.e species concentrations. In contrast, measurements for metabolic systems commonly concern fluxes, i.e. reaction rates [Costenoble et al., 2007].

Definition 9. The Hill variable is defined as

$$
m_{i, j}=K_{i, j}^{\eta_{i, j}}+c_{j}^{\eta_{i, j}}
$$

Theorem 10. Under Assumption 6 and 7 the reaction kinetic model (1) and (5) is equivalent to the parameter independent system

$$
\begin{aligned}
\frac{d}{d t} c_{i} & =\sum_{k=1}^{n_{r}} N_{i, k} r_{k} \\
\frac{d}{d t} m_{i, j} & =\eta_{i, j} c_{j}^{\eta_{i, j}-1} \sum_{k=1}^{n_{r}} N_{j, k} r_{k} \\
\frac{d}{d t} r_{i} & =r_{i} \sum_{j=1}^{n_{c}}\left(\left(\nu_{i, j} \frac{1}{c_{j}}-\frac{\eta_{i, j} c_{j}^{\eta_{i, j}-1}}{m_{i, j}}\right) \sum_{k=1}^{n_{r}} N_{j, k} r_{k}\right) .
\end{aligned}
$$

Proof. Hill variables: The dynamic description of $m$ is obtained by differentiation along the trajectory of (1)

$$
\frac{d}{d t} m_{i, j}=\eta_{i, j} c_{j}^{\eta_{i, j}-1} \dot{c}_{j} .
$$

Reaction rates: We multiply both sides of (5) with the denominator and take the logarithm

$$
\begin{aligned}
\sum_{j=1}^{n_{c}} \log \left(K_{i, j}^{\eta_{i, j}}+c_{j}^{\eta_{i, j}}\right) & +\log \left(r_{i}\right) \\
= & \log \left(\hat{r}_{i}\right)+\sum_{j=1}^{n_{c}} \nu_{i, j} \log \left(c_{j}\right) .
\end{aligned}
$$

Again taking the time derivative and using (6) yields

$$
\sum_{j=1}^{n_{c}} \frac{\dot{m}_{i, j}}{m_{i, j}}+\frac{\dot{r}_{i}}{r_{i}}=\sum_{j=1}^{n_{c}} \nu_{i, j} \frac{\dot{c}_{j}}{c_{j}} .
$$

Rearranging yields the differential equations for the reaction rates

$$
\dot{r}_{i}=r_{i}\left(\sum_{j=1}^{n_{c}} \nu_{i, j} \frac{\dot{c}_{j}}{c_{j}}-\sum_{j=1}^{n_{c}} \frac{\dot{m}_{i, j}}{m_{i, j}}\right) .
$$

The great advantage of this description is that the righthand-side does not depend on the parameters $\hat{r}_{i}$ and $K_{i, j}$ and that the output is linear (which follows directly from Assumption 7). By defining $x=\left[c^{T} \operatorname{vec}(m)^{T} r^{T}\right]^{T}(7)$ is of the form

$$
\frac{d}{d t} x=f(x), \quad x(0) \in \mathcal{M} \subset \mathbb{R}^{n}, \quad y=C \cdot x \in \mathbb{R}^{p} .
$$

The somewhat artificial introduction of additional states induces dependencies within the trajectories of the extended model. From (1) follows that the trajectories move on a manifold $\mathcal{M}$ of dimension $\leq n_{c}$, which is then, through the model extension, embedded in a state-space of dimension $n>n_{c}$. In Section 3.3 we will make use of the arising dependencies to infer the parameter values.

\subsection{Observer design}

For the reconstruction of all extended variables from time course measurements $y_{i}, i=1, \ldots, p$, a natural tool is provided by nonlinear observers. Assume that the system is trajectory observable and that we can transform it into observability normal form with $\bar{f}(z)=(Q \cdot f) \circ q^{-1}(z)$.

$$
\frac{d}{d t} z=\bar{f}(z), \quad z(0)=q\left(x_{0}\right) \in \mathcal{H}^{n}, \quad y=C \cdot z \in \mathbb{R}^{p} .
$$

Definition 11. A high gain observer with the gain parameter $\theta \in \mathbb{R}^{+}$in observer normal coordinates is of the form

$$
\frac{d}{d t} \tilde{z}=\bar{f}(\tilde{z})+L(\theta) \cdot[y-C \tilde{z}] .
$$

It consist of a simulation term $\bar{f}$ (a copy of the system) and a correction term $L \in \mathbb{R}^{n \times p}$ that feedbacks the error of measured $y$ and estimated output $\tilde{y}=C \tilde{z}$ and depends on a high gain-parameter $\theta$.

Some observers involve a state dependent correction term $L(x)$, whose design requires extensive symbolic manipulations that might not be feasible for large nonlinear systems [Birk and Zeitz, 1988]. Here, for simplicity, the correction term $L$ is designed independently of the actual states of the system taking a Lyapunov based approach [Gauthier et al., 1992]. Global convergence can be guaranteed if $\bar{f}$ is Lipschitz, whereby the Lipschitz constant can be interpreted as the maximal slope of $\bar{f}$ according to which the minimal observer gain has to be chosen. In the following, we extend the method for systems with multiple outputs $y_{k}, k=1, \ldots, p$.

The observer gain matrix $L$ is obtained by solving for each of the outputs $y_{k}$ the Lyapunov equations

$$
0=-\theta S_{\infty, k}-S_{\infty, k} A_{k}-A_{k}^{T} S_{\infty, k}+C_{k}^{T} C_{k},
$$

where $S_{\infty, k} \in \mathbb{R}^{k}, A_{k} \in \mathbb{R}^{n_{k} \times n_{k}}$ with $A_{i, j}=\delta_{i, j-1}{ }^{1}$, $C_{k}^{T} \in \mathbb{R}^{n_{k}}$ with $C_{k}=\delta_{1, k}$ and $n_{k}$ denotes the highest derivative of $y_{k}$ present in the observability map. The matrix $L \in \mathbb{R}^{n \times n}$ is then calculated with the blockdiagonal matrix of all inverse solutions

$$
L=\operatorname{diag}\left(S_{\infty, k}^{-1}\right) \cdot C^{T}, \quad C^{T} \in \mathbb{R}^{n \times p}, \quad C_{j, k}^{T}=\delta_{k, n_{k}} .
$$

Some additional calculation is necessary to obtain the observed states in the original (physically meaningful) coordinates. There are basically two possibilities:

(1) Transform the differential equations of the observer back into original coordinates using the inverse of the observability matrix $\frac{d}{d t} \tilde{x}=Q^{-1} \frac{d}{d t} \tilde{z}$. Then the observer is is a system of ordinary differential equations (proof see Appendix)

$$
\frac{d}{d t} \tilde{x}=f(x)+Q^{-1}(x) \cdot L \cdot[y-h(x)] .
$$

1 Here $\delta$ denotes the Kronecker symbol with $\delta_{i, j}=\left\{\begin{array}{ll}1 & \text { if } i=j \\ 0 & \text { if } i \neq j\end{array}\right.$. 
(2) Transform the observed trajectory back into original coordinates. Then the observer consists of a dynamic part in observability coordinates and an algebraic part [Vargas and Moreno, 2005]

$$
\begin{aligned}
\frac{d}{d t} \tilde{z} & =\bar{f}(\tilde{z})+L \cdot[y-C \tilde{z}], \\
\tilde{x} & =q^{-1}(\tilde{z}) .
\end{aligned}
$$

Remark 12. Calculation of the transformed system $\bar{f}$ and the inverse observability matrix $q^{-1}$ involves symbolic manipulation and might often be infeasible for large nonlinear systems. Considering that computational effort, the first observer strategy might be advantageous, particularly in the setting of a constant correction term $L$, where only the inverse of the observability matrix $Q^{-1}$ must be calculated.

A problem arises if local observability is lost somewhere along the observed trajectory. However, under certain conditions ( $q$ is globally injective with an uniformly continuous inverse), (11) is a so called $\epsilon$-approximate observer (see Vargas and Moreno [2005] for a detailed discussion). Clearly, observer (10) fails since $Q^{-1}$ does not exist where $\operatorname{det}(Q)=0$, but modifications are possible that result in so called event based observers [Vargas et al., 2003].

Definition 13. An event of duration $t_{\text {Event }}$ occurs if the inversion of $Q$ becomes ill conditioned during the observation process

$$
t_{\text {Event }}=\left\{t \in \mathbb{R}: \quad\left|\frac{\lambda_{\min }}{\lambda_{\max }}\right|<\delta\right\}
$$

where $\lambda_{\min }$ and $\lambda_{\max }$ are the absolute smallest and largest eigenvalue of $Q\left(x\left(t, x_{0}\right)\right)$ respectively, and $\delta$ is some predefined value $\delta>0$.

During an event, the determinant of $Q$ is close to zero, the inversion of $Q$ is numerically ill-conditioned and the correction term in (10) becomes very large. To resolve this issue, Vargas et al. [2003] proposed to switch the correction term $Q^{-1}(x) L$ in (10) to zero.

Proposition 14. [Vargas et al., 2003] For a trajectory observable system, the event based observer with sufficiently high gain $\theta$

$$
\frac{d}{d t} \tilde{z}=f(\tilde{z})+Q^{i n v}(\tilde{z}) L(\theta)(y-h(\tilde{z}))
$$

whereby

$$
Q^{i n v}=\left\{\begin{array}{ll}
Q^{-1} & \text { if }\left|\frac{\lambda_{\min }}{\lambda_{\max }}\right| \geq \delta, \\
Q^{\sim 1} & \text { if }\left|\frac{\lambda_{\min }}{\lambda_{\max }}\right|<\delta .
\end{array},\right.
$$

exhibits a limited error $x-\hat{x}$ if $\sum t_{\text {Event }, \mathrm{i}}<<t_{\text {end }}-t_{0}$.

Remark 15. For the example in Section 4 the above observer failed. Once trapped in an event, the system remained there due to the switch-off of the correction term.

\subsection{Inferring the parameters}

If all the extended states are known, for instance by applying one of the above discussed observers, the parameters can be estimated by means of a least-squares fit.

Definition 16. The estimation errors are defined as

$$
\begin{aligned}
\epsilon_{m_{i, j}}(t) & =m_{i, j}(t)-\left(K_{i, j}^{\eta_{i, j}}+c_{j}(t)^{\eta_{i, j}}\right) \\
\epsilon_{r_{i}}(t) & =r_{i}(t)-\hat{r}_{i} \prod_{j=1}^{n_{c}} \frac{c_{j}(t)^{\nu i, j}}{m_{i, j}(t)^{\bar{\eta}_{i, j}}} .
\end{aligned}
$$

Definition 17. The least squares cost functions are

$$
\Theta_{m_{i, j}}=\sum_{k=1}^{n_{t}}\left(\epsilon_{m_{i, j}}\left(t_{k}\right)\right)^{2}, \quad \Theta_{r_{i}}=\sum_{k=1}^{n_{t}}\left(\epsilon_{r_{i}}\left(t_{k}\right)\right)^{2} .
$$

Theorem 18. An optimal estimate for the parameters minimizing the cost functions (13) is

$$
\begin{aligned}
K_{i, j} & =\frac{1}{n_{t}} \sum_{k=1}^{n_{t}}\left(m_{i, j}\left(t_{k}\right)-c_{j}\left(t_{k}\right)\right), \\
\hat{r}_{i} & =\frac{\sum_{k=1}^{n_{t}} r_{i}\left(t_{k}\right) \chi(k)}{\sum_{k=1}^{n_{t}} \chi(k)}, \chi(k)=\prod_{j=1}^{n_{c}} \frac{c_{j}\left(t_{k}\right)^{\nu i, j}}{m_{i, j}\left(t_{k}\right)^{\bar{\eta}_{i, j}}} .
\end{aligned}
$$

Proof. We start with the sum of squared errors of the reaction rates

$$
\Phi_{r}=\frac{1}{n_{t}} \sum_{k=1}^{n_{t}}\left(r_{i}\left(t_{k}\right)-\hat{r}_{i} \prod_{j=1}^{n_{c}} \frac{c_{j}\left(t_{k}\right)^{\nu i, j}}{m_{i, j}\left(t_{k}\right)^{\bar{\eta}_{i, j}}}\right)^{2} .
$$

For the sake of simplicity, set $\chi\left(t_{k}\right)=\prod_{j=1}^{n_{c}} \frac{c_{j}\left(t_{k}\right)^{\nu i, j}}{m_{i, j}\left(t_{k}\right)^{\eta_{i, j}}}$, and expand the square

$$
\Phi_{r}=\frac{1}{n_{t}} \sum_{k=1}^{n_{t}}\left(r_{i}\left(t_{k}\right)^{2}-2 \hat{r}_{i}\left(t_{k}\right) r_{i} \chi\left(t_{k}\right)+\hat{r}_{i}^{2} \chi\left(t_{k}\right)^{2}\right) .
$$

Then the minimum is obtained by setting the partial derivative $\frac{\partial \Phi_{r}}{\partial \hat{r}_{i}}$ equal to zero

$$
\begin{aligned}
0 & =\frac{1}{n_{t}} \sum_{k=1}^{n_{t}}-2 r_{i}\left(t_{k}\right) \chi\left(t_{k}\right)+2 \hat{r}_{i} \chi\left(t_{k}\right)^{2} \\
& =\hat{r}_{i} \frac{2}{n_{t}} \sum_{k=1}^{n_{t}} \chi\left(t_{k}\right)^{2}-\frac{2}{n_{t}} \sum_{k=1}^{n_{t}} r_{i}\left(t_{k}\right) \chi\left(t_{k}\right) .
\end{aligned}
$$

Simple manipulation solves this for $\hat{r}_{i}$, resulting in (15).

A similar procedure for the sum of squares error of the Hill variables resulting in (14) is

$$
\begin{aligned}
& \frac{1}{n_{t}} \sum_{k}^{n_{t}}\left(m_{i, j}\left(t_{k}\right)-\left(K_{i, j}^{\eta_{i, j}}+c_{j}\left(t_{k}\right)^{\eta_{i, j}}\right)\right)^{2}= \\
& \frac{1}{n_{t}} \sum_{k}^{n_{t}}\left(m_{i, j}\left(t_{k}\right)^{2}-2 m_{i, j}\left(t_{k}\right)\left(K_{i, j}^{\eta_{i, j}}-c_{j}\left(t_{k}\right)^{\eta_{i, j}}\right)\right. \\
& \left.+\left(K_{i, j}^{\eta_{i, j}}+c_{j}\left(t_{k}\right)^{\eta_{i, j}}\right)^{2}\right) \stackrel{!}{=} \text { min. }
\end{aligned}
$$

Now we set the partial derivative to zero and solve for $K_{i, j}$

$$
\begin{aligned}
& \Rightarrow \quad \frac{1}{n_{t}} \sum_{k}^{n_{t}}\left(-2 m_{i, j}\left(t_{k}\right)+2\left(K_{i, j}^{\eta_{i, j}}+c_{j}\left(t_{k}\right)^{\eta_{i, j}}\right)\right)=0 \\
& \Leftrightarrow \quad K_{i, j}^{\eta_{i, j}}+\sum_{k}^{n_{t}}\left(-m_{i, j}\left(t_{k}\right)+c_{j}\left(t_{k}\right)^{\eta_{i, j}}\right)=0 \\
& \Leftrightarrow \quad K_{i, j}^{\eta_{i, j}}=\sum_{k}^{n_{t}}\left(m_{i, j}\left(t_{k}\right)-c_{j}\left(t_{k}\right)^{\eta_{i, j}}\right) .
\end{aligned}
$$

\section{EXAMPLE}

In order to provide a proof of concept for our approach, it is tested on the circadian clock of neuropsora modeled by a nonlinear feedback loop in the gene expression of the so called frequency protein (FRQ) [Leloup et al., 1999] 


\begin{tabular}{ccccccccc|c}
\multicolumn{7}{c|}{ Outputs \& derivatives used } & \\
$M$ & $F_{c}$ & $F_{n}$ & $r_{1}$ & $r_{2}$ & $r_{2^{\prime}}$ & $r_{3}$ & $r_{4}$ & $r_{5}$ & $\operatorname{rank}(Q)$ \\
\hline 0 & 1 & 2 & - & - & - & 1 & 1 & 1 & 12 \\
0 & 2 & 1 & - & - & - & 1 & 1 & 1 & 12 \\
1 & 0 & 2 & - & - & - & 1 & 1 & 1 & 11 \\
2 & 1 & 0 & - & - & - & 1 & 1 & 1 & 10 \\
1 & 1 & 1 & - & - & - & 1 & 0 & 2 & 10 \\
1 & 1 & 1 & - & - & - & 2 & 0 & 1 & 11 \\
3 & 3 & 3 & - & - & - & - & - & - & 11 \\
4 & 2 & 3 & - & - & - & - & - & - & 11 \\
4 & 3 & 2 & - & - & - & - & - & - & 12
\end{tabular}

Table 1. Selection of the observability analysis of the neurospora model, each row corresponds to one particular construction of $q$ with the entries being the $n_{i}-1$ in Equation 3. Observability is given if $Q=\frac{d q}{d x}$ has full rank $=12$.

$$
\begin{array}{lll}
\dot{M}=r_{3}-r_{5} & r_{1}=k_{s} M & r_{3}=v_{s} \frac{K_{1}^{4}}{K_{1}^{4}+F_{n}^{4}} \\
\dot{F}_{c}=r_{1}-r_{4}-r_{2}+r_{2^{\prime}} & r_{2}=k_{1} F_{c} & r_{4}=v_{d} \frac{F_{c}}{K_{d}+F_{c}} \\
\dot{F}_{n}=r_{2}-r_{2^{\prime}} & r_{2^{\prime}}=k_{2} F_{n} & r_{5}=v_{m} \frac{M}{K_{M}+M},
\end{array}
$$

where $M$ denotes the concentration of FRQ mRNA, $F_{c}$ and $F_{n}$ the concentration of FRQ protein in the cytosol and nucleus respectively, $r_{1}$ denotes the rate of translation, $r_{2}$ and $r_{2^{\prime}}$ of transport in and out the nucleus, $r_{3}$ of transcription, $r_{4}$ and $r_{5}$ of degradation.

The extended system is

$$
\frac{d}{d t}\left[\begin{array}{c}
M \\
F_{c} \\
F_{n} \\
m_{1} \\
m_{2} \\
m_{3} \\
r_{1} \\
r_{2} \\
r_{2^{\prime}} \\
r_{3} \\
r_{4} \\
r_{5}
\end{array}\right]=\left[\begin{array}{c}
r_{3}-r_{5} \\
r_{1}-r_{2}+r_{2^{\prime}}-r_{4} \\
r_{2}-r_{2^{\prime}} \\
2 F_{n}\left(r_{2}-r_{2^{\prime}}\right) \\
r_{1}-r_{2}+r_{2^{\prime}}-r_{4} \\
r_{3}-r_{5} \\
r_{1}\left(r_{3}-r_{5}\right) / M \\
\left.r_{2}-r_{2}+r_{2^{\prime}}-r_{4}\right) / F_{c} \\
r_{2^{\prime}}\left(r_{2}-r_{2^{\prime}}\right) / F_{n} \\
-2 r_{3} F_{n}\left(r_{2}-r_{2^{\prime}}\right) / m_{1} \\
r_{4}\left(r_{1}-r_{2}+r_{2^{\prime}}-r_{4}\right)\left(1 / F_{c}-1 / m_{2}\right) \\
r_{5}\left(r_{3}-r_{5}\right)\left(1 / M-1 / m_{3}\right)
\end{array}\right]
$$

For simplicity of the design, we assume in the following that the species concentrations as well as the transcription and degradation rates are measured [Shu and Hong-Hui, 2004]. (Note however, that the system is also observable if only the concentrations are measured $y=\left[M F_{c} F_{n}\right]$.)

$$
y=\left[\begin{array}{llllll}
M & F_{c} & F_{n} & r_{3} & r_{4} & r_{5}
\end{array}\right]^{T} .
$$

We explored different constructions of $q$, i.e. different combinations of chosen output derivatives, to analyze observability (Table 1). Thereby it is advisable to limit the oder of that derivatives for two reasons. First, to keep the observer design simple, and second to minimize numerical errors. An invertible $Q=\frac{\partial}{\partial x} q \in \mathbb{R}^{n \times n}$ is for example obtained using (Table 1 row 1 )

$$
q=\left[\begin{array}{llllllllllll}
M & F_{c} & \dot{F}_{c} & F_{n} & \dot{F}_{n} & \ddot{F}_{n} & r_{3} & \dot{r}_{3} & r_{3} & \dot{r}_{4} & r_{5} & \dot{r}_{5}
\end{array}\right]^{T} .
$$

However, there are singular points. Looking at the determinant tells us that local observability is lost if

$$
\begin{aligned}
r_{2}=r_{2^{\prime}} \vee r_{3}= & r_{5} \vee r_{1}+r_{2^{\prime}}=r_{2}+r_{4} \vee \\
& F_{c}\left(r_{2}-r_{2^{\prime}}\right)=F_{n}\left(r_{1}+r_{2^{\prime}}-r_{2}-r_{4}\right),
\end{aligned}
$$

and $q^{-1}$ is non-Lipschitz (see Appendix), but continuous under the image of $q$ since $q \circ q^{-1}=i d$.

Both observer structures, (10) and (11), were implemented. The limit for the condition number of Observer (10) is chosen to $\delta=10^{-4}$.

In a simulation study with the originally published parameters and the corresponding initial conditions for the extended system, the observers are initialized with $\tilde{x}_{i}(0)=$ $2 x_{i}(0)$ for all non-measured variables $i=4, \ldots, 9$. For the given initial condition, the event based observer fails (Figure 1a), whereas the $\epsilon$-approximative observer (15) converges (Figure 1b). There are periods where observer error increases due to the reduced observability properties of the system (Figure 1.3). Calculation of the parameters using the state estimates of (15) for $t>10 \mathrm{~h}$ to ensure convergence of the observer, does not exhibit errors $>0.5 \%$ (Table 4), proving the applicability of the method. Note that biological measurements usually exhibit errors about one order of magnitude larger or worse.

\section{CONCLUSIONS}

This paper proposes a novel method for kinetic parameter estimation that is particularly tailored to biological models consisting of ordinary differential equations. Basically, the potential of the presented method lies in the model extension, which establishes a one-to-one correspondence between parameters and states. Therewith, the main task of the method becomes to reconstruct the extended trajectory from the measurements. Here, we used Lyapunov based observers, however, since biological data is usually discrete and noisy, other techniques might be useful, such as Kalman filters or hybrid observers composed of a continuous simulation part $f(x)$ updated at discrete timestamps. The inherent dependencies of the observed trajectory given by the definition of the extended states allows us to solve a least squares criterion for the parameters. Note that estimation of the parameters could in principle also be achieved by identifying the true initial conditions of the observer, which is however a hard problem. In addition, the benefits that might arise from the extended system being parameter independent are not yet fully explored.

\section{REFERENCES}

Anguelova, M., G. Cedersund, M. Johansson, C. Franzén and Wennberg, B. (2007). Conservation laws and unidentifiability of rate expressions in biochemical models. IET Sys. Biol. 1(4), 230-237.

Audoly, S., G. Bellu, L. D’Angio, M. Saccomani and Cobelli, C. (2001). Global identifiability of nonlinear

\begin{tabular}{l|ccc} 
Max. rates & $k_{s}$ & $k_{1}$ & $k_{2}$ \\
\hline True & 0.5000 & 0.5000 & 0.6000 \\
Obs. (11) & 0.4999 & 0.4988 & 0.5985 \\
Hill const. & $K_{1}$ & $K_{d}$ & $K_{m}$ \\
\hline True & 0.5000 & 0.1300 & 0.5000 \\
Obs. (11) & 0.4988 & 0.1300 & 0.5048
\end{tabular}

Table 2. True and estimated parameter values. Units are $k_{i}\left(\mathrm{~h}^{-1}\right), v_{i}\left(\mathrm{nMh}^{-1}\right), K_{i}(\mathrm{nM})$. 

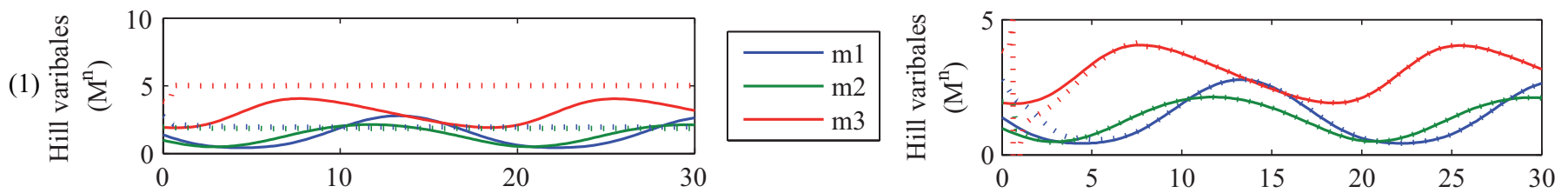

(2)
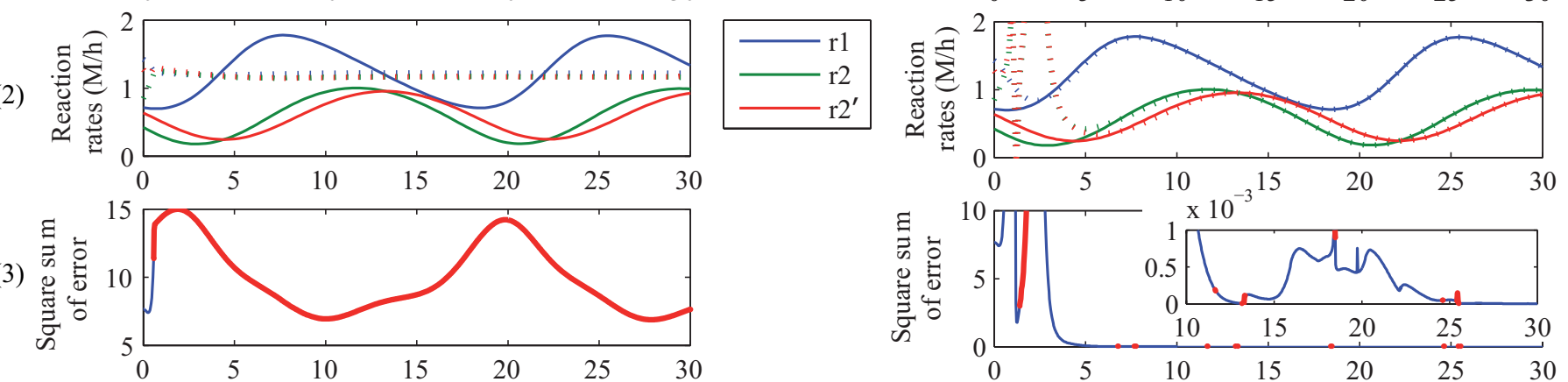

(a) Time (h)

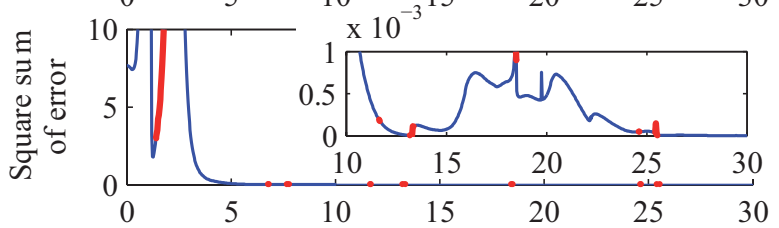

(b) Time (h)

Fig. 1. (1) - (2) The real (solid) and estimated (dotted) trajectories of the non measured states for (a) the event based observer (10) and (b) the $\epsilon$-approximative observer (11) with gain parameter $\theta=1$. (3) The sum of squares error of the observers $\sum_{j}^{n}\left(x_{j, \text { est }}-x_{j \text {,real }}\right)^{2}$. The red lines indicate where events occur on the observed trajectory. The inlay shows a magnification for $t>10 \mathrm{~h}$.

models of biological systems. Biomedical Engineering, IEEE Transactions on 48(1), 55-65.

Birk, J. and Zeitz, M. (1988). Extended luenberger observer for non-linear multivariable systems. International Journal of Control 47, 1823-1836.

Costenoble, R. et al. (2007). 13c-labeled metabolic flux analysis of a fedbatch culture of elutriated saccharomyces cerevisiae. FEMS Yeast Res 7(4), 511-526.

Gauthier, J., H. Hammouri and Othman, S. (1992). A simple observer for nonlinear systems applications to bioreactors. Automatic Control, IEEE Transactions on 37(6), 875-880.

Leloup, J. C., D. Gonze and Goldbeter, A. (1999). Limit cycle models for circadian rhythms based on transcriptional regulation in drosophila and neurospora. J Biol Rhythms 14(6), 433-448.

Moles, C. G., P. Mendes and Banga, J. R. (2003). Parameter estimation in biochemical pathways: a comparison of global optimization methods. Genome Res 13(11), 2467-2474.

Peifer, M. and Timmer, J. (2007). Parameter estimation in ordinary differential equations for biochemical processes using the method of multiple shooting. IET Syst Biol $\mathbf{1}(2), 78-88$.

Shu, Y. and Hong-Hui, L. (2004). Transcription, translation, degradation, and circadian clock. Biochemical and Biophysical Research Communications 321(1), 1-6.

Vargas, A., J. Moreno and Zeitz, M. (2003). Event-based observer design for observable nonlinear systems with bad input points. In: CD Proceedings European Conference of Control, Cambridge/UK.

Vargas, A. and Moreno, J. A. (2005). Approximate highgain observers for non-Lipschitz observability forms. International Journal of Control 78, 247-253(7).

Voit, E. O. and Almeida, J. (2004). Decoupling dynamical systems for pathway identification from metabolic profiles. Bioinformatics 20(11), 1670-1681.

\section{Appendix A. (BACK-)TRANSFORMATION FROM} THE OBSERVER INTO ORIGINAL COORDINATES

Consider a continuous observer in observer normal form [Birk and Zeitz, 1988]

$$
\frac{d}{d t} \tilde{z}=\bar{f}(\tilde{z})+L(y-\tilde{y}),
$$

with a constant gain matrix $L$. We transform back into original coordinates by differentiating $\tilde{x}=q^{-1}(\tilde{z})$.

$$
\frac{d}{d t} \tilde{x}=\frac{d q}{d \tilde{z}} \frac{d \tilde{z}}{d t}=\left[Q^{-1}(\tilde{z}) g(\tilde{z})+Q^{-1}(\tilde{z}) L(y-\tilde{y})\right]_{\tilde{z}=q(\tilde{x})} .
$$

Remind that $f=Q^{-1} g \circ q$, wherewith follows

$$
\frac{d}{d t} \tilde{x}=f(\tilde{x})+Q^{-1}(\tilde{x}) L(y-\tilde{y}) .
$$

\section{Appendix B. INVERSE OBSERVABILITY MAP OF THE NEUROPORA MODEL}

$$
q^{-1}=\left[\begin{array}{c}
z_{1} \\
z_{4} \\
z_{2} \\
-2 z_{7} z_{4} z_{5} / z_{8} \\
-z_{9} z_{2} z_{3} /\left(z_{10} z_{2}-z_{9} z_{3}\right) \\
z_{11} z_{1}\left(z_{11}-z_{7}\right) /\left(-z_{11} z_{7}+z_{11}^{2}+z_{12} z_{1}\right) \\
z_{5}+z_{9}+z_{3} \\
-z_{4}\left(-z_{5} z_{3}+z_{6} z_{2}\right) /\left(-z_{4} z_{3}+z_{2} z_{5}\right) \\
-z_{2}\left(z_{6} z_{4}-z_{5}^{2}\right) /\left(-z_{4} z_{3}+z_{2} z_{5}\right) \\
z_{7} \\
z_{9} \\
z_{11}
\end{array}\right]
$$

